\title{
Clinical, Laboratory Characteristics and Pregnancy Outcome of COVID-19 Patients Admitted in the Largest COVID Dedicated Hospital of Bangladesh
}

\author{
S. K. Jakaria Been Sayeed ${ }^{1, ~ *, ~ M d . ~ M u j i b u r ~ R a h m a n ~}{ }^{1}$, A. K. M. Humayon Kabir ${ }^{1}$, \\ Md. Moniruzzaman ${ }^{2}$, Reaz Mahmud ${ }^{1}$, Mohammad Abdullah Yusuf ${ }^{2}$, Sabrina Rahman ${ }^{1}$ \\ ${ }^{1}$ Medicine Department, Dhaka Medical College Hospital, Dhaka, Bangladesh \\ ${ }^{2}$ Stroke Unit, National Institute of Neurosciences \& Hospital, Dhaka, Bangladesh
}

Email address:

skjakaria1@gmail.com (S. K. J. B. Sayeed), mmrahman61@gmail.com (Md. M. Rahman), drakmhkabir@gmail.com (A. K. M. H. Kabir), dr.monirsomc41@gmail.com (Md. Moniruzzaman), reazdmc22@yahoo.com (R. Mahmud), ayusuf75@yahoo.com (M. A. Yusuf), rahmansabrina20@gmail.com (S. Rahman)

${ }^{*}$ Corresponding author

\section{To cite this article:}

S. K. Jakaria Been Sayeed, Md. Mujibur Rahman, A. K. M. Humayon Kabir, Md. Moniruzzaman, Reaz Mahmud, Mohammad Abdullah Yusuf, Sabrina Rahman. Clinical, Laboratory Characteristics and Pregnancy Outcome of COVID-19 Patients Admitted in the Largest COVID Dedicated Hospital of Bangladesh. American Journal of Internal Medicine. Vol. 9, No. 1, 2021, pp. 11-16.

doi: 10.11648/j.ajim.20210901.12

Received: December 18, 2020; Accepted: December 30, 2020; Published: January 15, 2021

\begin{abstract}
Background: The 2019 novel corona virus (SARS-CoV-2) has become pandemic, it is of paramount importance to conduct near-real-time surveillance of women who are hospitalized and test positive for COVID-19 during pregnancy. Objective: The purpose of the present study was to evaluate clinical and laboratory characteristics and pregnancy outcome among COVID-19 patients. Methodology: This was a prospective longitudinal study done in COVID dedicated unit of Dhaka Medical College Hospital. All pregnant women admitted with confirmed COVID-19 were included. Result: Among 68 pregnant COVID patients mean (SD) age was 26.58 (4.30) years where gestational age was 25.90 (5.34) weeks. Most common symptoms were fever (94\%), cough (79\%), sore throat (59\%), shortness of breath (44), myalgia (34\%). According to severity assessment, 51 (75\%) was found to be mild, moderate 9 (13\%) and severe 8 (12\%) respectively. Regarding overall maternal outcome among 68 patients 64 (94\%) recovered and discharged, 2 (3\%) of them recovered but abortion occurred and 2 (3\%) died. Mean (SD) gestational age during delivery was 37.8 (1.2) weeks. Vaginal delivery was done in 18 (35\%), cesarean section in $33(65 \%)$ and $13(19 \%)$ continuing pregnancy. Among fifty one neonate, only $41(80 \%)$ completed RT-PCR test and found negative. Regarding neonatal outcome, 48 (94.2\%) term baby, $3(5.8 \%)$ preterm, neonatal pneumonia $1(1 \%)$, neonatal hyperbilirubinemia $3(6 \%)$ were observed. Conclusion: The SARS-CoV-2 infection during pregnancy might not associate with adverse obstetrical and neonatal outcomes. It appears to be unlikely of perinatal transmission of SARS-CoV-2.
\end{abstract}

Keywords: SARS-CoV-2, COVID-19, Pregnancy, Outcome

\section{Introduction}

The 2019 novel corona virus (SARS-CoV-2) has become pandemic, first reported from Wuhan City of Hubei Province of China [1]. Transmission can occur through respiratory droplets from coughing and sneezing, however, aerosol transmission is also possible [2]. Clinical spectrum of COVID-19 varies from asymptomatic or symptomatic forms to clinical conditions like respiratory failure and multiple organ dysfunction syndromes (MODS) [3]. Most common clinical manifestations are fever, fatigue, cough and expectoration. Other symptoms were myalgia, anorexia, chest tightness, shortness of breath, nausea and vomiting, diarrhea, headache, and abdominal pain [4]. There are 
numerous differences with significant correlation in laboratory findings including neutrophil and lymphocyte count, as well as levels of plasma glucose, aspartate aminotransferase (AST), alanine aminotransferase (ALT), lactate dehydrogenase (LDH), D-dimer, erythrocyte sedimentation rate (ESR), C Reactive protein (CRP), Ferritin and procalcitonin (PCT) [5]. However, during pregnancy changes occurs in respiratory, endocrine, cardiovascular system due to changes in hormones and physiological structure [6]. Moreover, cell mediated immunity is suppressed, that might make pregnant women more prone to critical illness after infected with the virus [7]. It is of paramount importance to know about clinical characteristics, biochemical, radiological pictures of COVID-19 and outcome among pregnant patients that might help in management especially in a resource limited country like Bangladesh. So, we conducted this study.

\section{Methodology}

A prospective longitudinal study was done in COVID-19 dedicated unit of Dhaka Medical College Hospital from March 15, 2020 to August 15, 2020. All pregnant women admitted with symptoms suggestive of COVID-19 followed by confirmation with RT-PCR for SARS-CoV-2 were included. For case definitions and clinical severity assessment WHO [8] and National Guideline of Bangladesh for COVID-19 [9] were followed. Consecutive and purposive sampling techniques were used. Maternal outcome were defined by presence of sepsis, severe pneumonia, abortion, preterm labor, preeclampsia, GDM (Gestational Diabetes Mellitus), post or ante partum hemorrhage and death. However, neonatal outcome were assessed by birth weight, congenital anomaly, birth asphyxia, neonatal pneumonia, diarrhea, hyperbilirubinemia and hypoglycemia. Vital parameters with oxygen saturation were checked regularly. Laboratory investigations like Complete blood count, CRP (C-Reactive Protein), Ferritin, RBS (Random Blood Sugar), Creatinine, ALT (Alanine Transaminase), APTT (Activated Partial Thromboplastin Time), D-dimer, electrolyte, CT scan chest (if possible) and ECG were done. Ultra sonogram of pregnancy profile with anomaly scan performed after one month of discharge to assess congenital anomaly, intrauterine growth retardation (IUGR). Majority of the neonate had done with RT-PCR for SARS-CoV-2 (nasopharynx) test within 24 hours of delivery. Regular follow up was done by social media like messenger or what's app video session every one month later after discharge up to delivery. Data were analyzed by SPSS 23 version. Measurement data of normal distribution was expressed as mean \pm standard deviation. All counts and measurement data were descriptive statistics, and the results were expressed as mean, median \&percentages. Chi square $\chi 2$ was used for categorical variables. Informed consent and ethical approval were taken accordingly (Protocol numberMEU-DMC/ECC/2020/21).

\section{Results}

Total 68 pregnant COVID-19 patients were included in this study. Mean (SD) age was 26.58 (4.30) years; where gestational age 26 (5) weeks. Most of them (88\%) were residing in urban area and $30(44 \%)$ had positive history of contact with suspected or confirmed COVID-19 patients. Mean body mass index was $22.7 \mathrm{~kg}$ per $\mathrm{m}^{2}$ (5.4) Common co-morbidities were diabetes mellitus 13 (19\%), hypertension $6(9 \%)$ and bronchial asthma 5 (7\%) (Table 1) Median duration of symptoms were 7 days (range 5-10) where symptomatic improvement required 18 days (range 13-21) from onset of disease. Most common symptoms were fever 64 (94\%), cough 54 (79\%), sore throat $40(59 \%)$, shortness of breath $30(44 \%)$, myalgia 23 (34\%), diarrhea $20(29 \%)$ and vomiting 18 (26\%). Hypoxia and hypotension were present in $36(53 \%)$ and $10(15 \%)$ cases respectively. Moreover, according to severity assessment, 51 (75\%) was found to be mild, moderate $9(13 \%)$ and severe $8(12 \%)$ (Table 2). Complete blood count revealed neutrophilia in 41 $(60 \%)$ cases, lymphopenia in 30 (44\%) cases, thrombocytopenia in 27 (39\%), low hemoglobin in $4(\%)$ cases. Raised C-Reactive Protein (CRP), D-dimer, APTT and Ferritin was found in 63 (56\%), 28 (42\%), 17 (25\%) and $64(94 \%)$ cases respectively. Alanine aminotransferase (ALT) was above the normal range in 30 (44\%) cases, Random blood sugar raised in $17(25 \%)$ cases, serum creatinine raised in $6(9 \%)$ cases during admission while almost all of these values came to back to normal range during discharge (Table 2). Only 13 patients (moderate to severe) had undergone high resolution CT scan chest where unilateral and bilateral pneumonia found in $5(7 \%)$ and 8 (11\%) cases respectively. Regarding overall maternal outcome among 68 patients, 64 (94\%) recovered and discharged, 2 (3\%) of them recovered but abortion occurred and $2(3 \%)$ died in the hospital. However, adverse pregnancy outcome like sepsis in $7(10 \%)$, severe pneumonia in $8(12 \%)$, preterm labor in $3(4 \%)$, abortion in $2(3 \%)$, gestational diabetes mellitus in $4(6 \%)$, preeclampsia in $2(3 \%)$, postpartum hemorrhage in $2(3 \%)$ and death occurred in $2(3 \%)$ cases. Vaginal delivery was done in $18(36 \%)$, cesarean section in $33(65 \%)$ and $13(19 \%)$ continuing pregnancy till writing this report (Table 3). Neonatal outcome was good, 48 (94\%) were term baby, 3 $(6 \%)$ preterm. Median birth weight was $2.52(2.1-3.4) \mathrm{kg}$. Only $3(6 \%)$ suffered from neonatal hyperbilirubinemia and $1(2 \%)$ neonatal pneumonia. Among 51 neonates, 41 (80\%) completed RT-PCR test and found negative but rest of them did not complete the test (Table 4). 
Table 1. General Information and Co-morbidities of 68 pregnant women with COVID-19.

\begin{tabular}{ll}
\hline Trait & Mean $( \pm$ SD), Frequency $\mathbf{~}(\%)$ \\
\hline Age & $27( \pm 4)$ years \\
Gestational age at illness & $26( \pm 5)$ weeks \\
Gestational age at delivery & $37( \pm 1)$ weeks \\
History of contact & $30(44)$ \\
Body Mass Index & 22.7 kg per m ${ }^{2}(5.4)$ \\
Residency & \\
Urban & $60(88)$ \\
Rural & $8(12)$ \\
Co-morbidities & $13(19)$ \\
Diabetes mellitus & $6(9)$ \\
Hypertension & $5(7)$ \\
Asthma & $1(1)$ \\
Rheumatic heart disease & $1(1)$ \\
Heart failure & $1(1)$ \\
Systemic lupus erythematosus & \\
\hline
\end{tabular}

Table 2. Clinical characteristics and laboratory findings of 68 pregnant women with COVID-19.

\begin{tabular}{|c|c|}
\hline Trait & Median (Range), Mean $( \pm$ SD) frequency N $(\%)$ \\
\hline Duration of symptoms & $7(5-10)$ days \\
\hline Duration of Recovery & $17(13-21)$ days \\
\hline \multicolumn{2}{|l|}{ Signs \& Symptoms } \\
\hline Fever & $64(94)$ \\
\hline Cough & $54(79)$ \\
\hline Sore Throat & $40(59)$ \\
\hline Sputum production & $6(8)$ \\
\hline Shortness of breath & $30(44)$ \\
\hline Chest pain & $5(7)$ \\
\hline Diarrhea & $20(29)$ \\
\hline Vomiting & $18(26)$ \\
\hline Myalgia & $23(34)$ \\
\hline Anosmia & $19(28)$ \\
\hline Tachypnea & $36(53)$ \\
\hline Hypotension & $10(15)$ \\
\hline Hypoxia & $36(53)$ \\
\hline $\mathrm{SpO} 2$ during admission on air & $91( \pm 8)$ \\
\hline \multicolumn{2}{|l|}{ Clinical type } \\
\hline Mild & $51(75)$ \\
\hline Moderate & $9(13)$ \\
\hline Severe & $8(12)$ \\
\hline \multicolumn{2}{|l|}{ Laboratory findings } \\
\hline Neutrophilia & $41(60)$ \\
\hline Lymphopenia & $30(44)$ \\
\hline Thrombocytopenia & $27(40)$ \\
\hline Raised CRP (N= 0-10 ng/ml) & $63(93)$ \\
\hline Raised ALT $(\mathrm{N}=10-40 \mathrm{IU} / \mathrm{L})$ & $30(44)$ \\
\hline Raised Ferritin $(\mathrm{N}=10-120 \mathrm{ng} / \mathrm{ml})$ & $64(94)$ \\
\hline Raised D-dimer $(\mathrm{N}=<500 \mathrm{ng} / \mathrm{ml})$ & $28(42)$ \\
\hline Raised APTT $(\mathrm{N}=30-40 \mathrm{sec})$ & $17(25)$ \\
\hline Hyperglycemia $(\mathrm{N} \leq 11.1 \mathrm{mmol} / \mathrm{l})$ & $17(25)$ \\
\hline Raised S. Creatinine $(\mathrm{N}=0.5-1.1 \mathrm{mg} / \mathrm{dl})$ & $6(9)$ \\
\hline Hyponatremia $(\mathrm{N}=132-137 \mathrm{mmol} / \mathrm{l})$ & $14(21)$ \\
\hline
\end{tabular}

APTT- Activated Partial Thromboplastin Time, CRP- C Reactive protein, ALT (Alanine Transaminase)

Table 3. Outcome among 68 pregnant women with COVID-19.

\begin{tabular}{ll}
\hline Trait & Frequency N (\%) \\
\hline Pregnancy Complications & $7(10)$ \\
Sepsis & $8(12)$ \\
Severe Pneumonia & $2(3)$ \\
Abortion & $3(4)$ \\
Pre Term Labor & $3(4)$ \\
Low birth weight & $2(3)$ \\
Pre-eclampsia & $4(6)$ \\
\hline Gestational DM & \\
\hline
\end{tabular}




\begin{tabular}{ll}
\hline Trait & Frequency N (\%) \\
\hline Post-partum hemorrhage & $2(3)$ \\
Fetal Distress & $7(10)$ \\
Maternal Death & $2(3)$ \\
Mode of Delivery & \\
Vaginal & $18(35)$ \\
Caesarian Section & $33(65)$ \\
Pregnancy Outcome & \\
Recovered with good outcome & $51(75)$ \\
Recovered with pregnancy ongoing & $13(19)$ \\
Death & $2(3)$ \\
\hline
\end{tabular}

Table 4. Neonatal Outcomes

\begin{tabular}{ll}
\hline Trait & Frequency N (\%), Median (Range) \\
\hline Neonatal Outcome & $3(6)$ \\
Preterm Baby & $48(94)$ \\
Full term baby & \\
Birth weight $(\mathrm{Kg})$ & $2.5(2.1-3.4)$ \\
Median (Ref-2.5-3.5 kg) & $1(2)$ \\
Neonatal Complication & $3(6)$ \\
Neonatal pneumonia & \\
Neonatal hyperbilirubinemia & 0 \\
RT-PCR for SARS-CoV-2 & $41(80)$ \\
Positive & $10(20)$ \\
Negative & \\
Not done &
\end{tabular}

\section{Discussion}

In this prospective cohort study, 68 pregnant COVID-19 patients were admitted at COVID unit of Dhaka Medical College Hospital. Majority of them were residing in urban area and $30(44 \%)$ had positive history of contact with suspected or confirmed COVID-19 patients. Mean gestational age on admission was 26 (5.34) weeks, Median duration of symptoms were 7 days (range 5-10) where symptomatic improvement required 18 days (range 13-21) from onset of disease. In our study, most common symptoms were fever, cough, sore throat, shortness of breath, myalgia, diarrhea, vomiting, anosmia that were very much similar with Zhang L, et al. [10] \& Huang C, Wang Y, Li X, et al. [11]. Majority (75\%) of pregnant women had mild symptoms with stable respiratory function where only $25 \%$ had moderate to severe symptoms evidenced by presence of hypoxia, tachypnea, hypotension with derangement of blood and biochemical markers, similar to Zaigham M, Andersson [12] but different from San-Juana R et al. [13], described only $40 \%$ had mild disease. In this study, 47 patients terminated pregnancy at 3 rd trimester and the gestational age was ranging from 36 to 39 weeks. Abortion occurred in two cases around 13+ weeks. 33 cases were terminated by cesarean section, only few of those due to preterm labor, severe preeclampsia, fetal distress, history of previous cesarean section, severe COVID-19 and mostly patients own interest. Zhang L, et al. [10] described similar findings but sample size and gestational age at delivery was different. One of the reasons of behind increased caesarean section at $37+$ weeksmay be due to social and psychosocial factors related to COVID-19. Patients who undergone vaginal delivery had mild disease and improved early but those had cesarean delivery took more days to recovery; three of them admitted to ICU and recovered eventually. In a single center study in Spain, Martínez-Perez O, Vouga M, Cruz Melguizo S, et al [14] described similarly though sample size was different. Under strict isolation and active treatment, majority $64(94 \%)$ patients had been recovered and discharged. However, two patients died, both of them had severe COVID-19 (Sepsis \& ARDS) with chronic disease like heart failure with chronic rheumatic heart disease and systemic lupus erythematosus (SLE). Patient who had heart failure was not under regular treatment and patient who had SLE lost to follow up for last 6 months. These factors may be the contributing factor along with COVID-19 for their severity and death. Spontaneous abortion occurred in two patients who were primi with a gestational age around 15-17 weeks whom don't have any co-morbidity. Still we don't know whether it was due to COVID-19, Zhang L, et al [10] had also observed that but could not make any conclusive comments regarding COVID-19 in relation to abortion.

Regarding blood picture showed Neutrophilia, Ferritin, APTT and D-dimer all were raised in variable percentage in our study, similar findings observed by Zhang L, et al. [10], Zaigham M, Andersson [13] and Vlachodimitropoulou Koumoutsea E et al. [15]. Only 13 patients in this study undergone HRCT chest scan, had typical HRCT manifestations like multiple flaky, patchy, segmental ground glass shadows in one or both lungs, mainly distributed outside the band similar to the findings mentioned by Lei J, et al. [16]. Among 68 pregnant COVID-19 we found 28 (42\%) had raised D-dimer, raised APTT in 17 (25\%), thrombocytopenia in 27 (40\%) patients, 
Vlachodimitropoulou Koumoutsea E et al [15] observed similar findings. We kept 51 pregnant patients in close monitoring for variable duration who delivered 51 healthy baby, did RT-PCR for SARS-CoV-2 within 24 hours of delivery in $41(80 \%)$ neonates, all of them found negative, but for rest of the neonates test were not done due to parents inconvenience. Still our observation is vertical transmission from mother to fetus very unlikely though we did the test only from nasopharyngeal swab. No nucleic acid tests were performed on breast milk, placenta, amniotic fluid, and cord blood of pregnant women. Martínez-Perez O, Vouga M, Cruz Melguizo S et al [14] described 3 (4\%) of 72 newborns tested within 6 hours after birth had a positive test result in RT-PCR. However, repeat testing at 48 hours was negative. None developed COVID-19 symptoms within 10 days. However, they did not mention clearly whether it was due to vertical transmission. Although Zhang L, et al [10] analyzed 18 neonates delivered by 18 pregnant COVID-19 ladies, but none of them found to be positive for SARS-CoV-2. Regarding neonatal outcome, 48 (94\%) were term baby having normal birth weight though $3(6 \%)$ had low birth weight only $3(6 \%)$ suffered neonatal hyperbilirubinaemia, 1 (2\%) neonatal pneumonia. Probably, reason behind that their mothers were malnourished. Our observation showed favorable outcome of neonates among pregnant COVID-19 women. Zhang L, et al [10], Martínez-Perez O, Vouga M, Cruz Melguizo S et al [14] also found more or less favorable neonatal outcome with few exception. A systematic review by Zaigham M, Andersson [12] also described favorable neonatal outcome.

\section{Limitations}

However, we do have certain limitations like lack of sufficient information on newborns to determine vertical transmission, sample size was small and lack of association between cesarean delivery and risk of ICU admission.

\section{Conclusion}

In conclusion, in our study adverse outcome due to SARS$\mathrm{CoV}-2$ infection during pregnancy has been observed in very few patients. It appears to be unlikely of perinatal transmission of SARS-CoV-2, however, large scale studies with adequate samples are needed to rule out the potential risk of vertical transmission of SARS-CoV-2 and adverse pregnancy outcome.

\section{Declaration of Conflict of Interest}

All the authors do not have any possible conflicts of interest.

\section{Acknowledgements}

Special thanks to Prof. Salma Rouf, Prof. AKM Shamsuzzaman, Dr. Farhana Binte Monayem, Dr. Md.
Khairul Islam, Dr. Nandalal Sutradhar, Dr. Md. Nazmul Islam, Dr. Istiaque Ahmed, Dr. Md. Nazim Uddin, Dr. Mizanur Rahman, Dr. Haniful Haque, Dr. Tasneem Ahmed, Department of Gynaecology \& Obstetrics and Department of Paediatrics, Dhaka Medical College Hospital for data collection, maintaining follow up schedule \& providing excellent support throughout study period. I am acknowledging all of my patients who gave their consent for participation in this study.

\section{References}

[1] Guan W, Ni Z, Hu Y, et al. Clinical characteristics of corona virus disease 2019 in China. N Engl J Med 2020; 382: 17081720. 10.1056/NEJMoa2002032.

[2] Guo ZD, Wang ZY, Zhang SF, et al. Aerosol and Surface Distribution of Severe Acute Respiratory Syndrome Corona virus 2 in Hospital Wards, Wuhan, China, 2020. Emerging Infect. Dis. 2020; $26 \quad$ (7): 1583 1591.10.3201/eid2607.200885.

[3] Cascella M, Rajnik M, Cuomo A, et al. Features, Evaluation and Treatment Corona virus (COVID-19) [Updated $2020 \mathrm{Jul}$ 4]. In: Stat Pearls [Internet]. Treasure Island (FL): Stat Pearls Publishing; 2020 Jan-. Available from: https://www.ncbi.nlm.nih.gov/books/NBK554776.

[4] Zhu J, Ji P, Pang J, et al. Clinical characteristics of 3062 COVID-19 patients: A meta analysis. J Med Virol. 2020.92: 1902-1914. 10.1002/jmv.25884.

[5] Wang D, Li FR, Wang J, et al. Association between severity of COVID-19 and clinical and biochemical characteristics: a cross-sectional Study, Research square: infectious disease [preprint]. 10.21203/rs.3.rs-18482/v1.

[6] Contreras G, Gutiérrez M, Beroíza T, et al. Ventilatory drive and respiratory muscle function in pregnancy. Am Rev Respir Dis. 1991; 144: 837-41. https://doi.org/10.1164/ajrccm/144.4.

[7] Lederman MM. Cell-mediated immunity and pregnancy [J]. Chest. 1984; 86: 6S-9S.https://doi.org/10.1186/s12884-02003026-3.

[8] WHO guidance on management of severe acute respiratory infection (SARI) when COVID19 is suspected; https://www.who.int/publications-detail/clinical-managementof-severe-acute-respiratory-infection-when-novelcoronavirus-(ncov)-infection-is-suspected.

[9] National Guidelines on Clinical Management of Coronavirus Disease 2019 (Covid-19), 27 May, 2020 https://dghs.gov.bd/images/docs/Guideline/COVID_Guideline .pdf.

[10] Zhang, L., Dong, L., Ming, L. et al. Severe acute respiratory syndrome coronavirus 2 (SARS-CoV-2) infections during late pregnancy: a report of 18 patients from Wuhan, China. BMC $\begin{array}{llll}\text { Pregnancy Childbirth 20, } 394 \quad \text { (2020). } & \text { 20 }\end{array}$ https://doi.org/10.1186/s12884-020-03026-3.

[11] Huang C, Wang Y, Li X, et al. Clinical features of patients infected with 2019 novel coronavirus in Wuhan, China. Lancet 2020; 395: 497-506. https://doi.org/10.1016/S01406736(20)30183-5. 
[12] Zaigham M, Andersson O. Maternal and perinatal outcomes with COVID-19: A systematic review of 108 pregnancies. Acta Obstet Gynecol Scand. 2020; 99 (7): 823-829. 10.1111/aogs.13867.

[13] San-Juana R, Barberob P, Fernandez-Ruiz M, et al. Incidence and clinical profiles of COVID-19 pneumonia in pregnant women: A single-centre cohort study from Spain. Eclinical medicine. 2020; 23 : 100407. https://doi.org/10.1016/j.eclinm.2020.100407.

[14] Martínez-Perez O, Vouga M, Cruz Melguizo S, et al. Association Between Mode of Delivery Among Pregnant Women With COVID-19 and Maternal and Neonatal
Outcomes in Spain [published online ahead of print, 2020 Jun 8] [published correction appears in JAMA. 2020 21; 324 (3): 305]. JAMA. 2020; e2010125. 10.1001/jama.2020.10125.

[15] Vlachodimitropoulou Koumoutsea E, Vivanti AJ, Shehata N, et al. COVID-19 and acute coagulopathy in pregnancy. J Thromb Haemost. 2020; 18: 1648-1652.10.1111/jth.14856.

[16] Lei J, Li J, Li X, et al. CT Imaging of the 2019 novel coronavirus (COVID-19) pneumonia [J]. Radiology. 2020; 31: 200236. https://doi.org/10.1148/radiol.2020200236. 